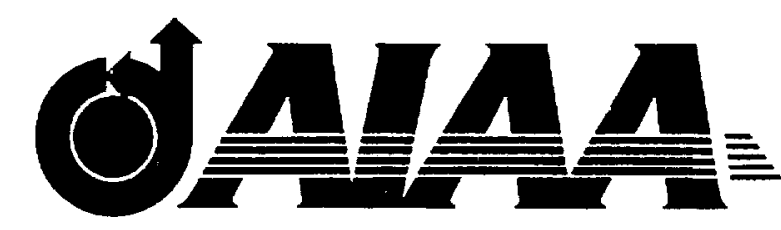

\title{
AIAA 98-0083 \\ Evaluation of turbulence-model performance as applied to jet-noise prediction
}

S. L. Woodruff

Florida State University, Tallahassee FL 32306-4120

J. M. Seiner

Langley Research Center, National Aeronautics and

Space Administration, Hampton, Virginia 23681

M. Y. Hussaini

Florida State University, Tallahassee FL 32306-4120

G. Erlebacher

Florida State University, Tallahassee FL 32306-4120

\section{6th Annual Aerospace Sciences Meeting and Exhibit January 12-15, 1998/Reno, NV}





\title{
Evaluation of turbulence-model performance as applied to jet-noise prediction
}

\author{
S. L. Woodruff* \\ Florida State University, Tallahassee FL 32306-4120
}

J. M. Seiner ${ }^{\dagger}$
Langley Research Center, National Aeronautics and Space Administration, Hampton, Virginia 23681

M. Y. Hussaini ${ }^{\ddagger}$

Florida State University, Tallahassee FL 32306-4120

G. Erlebacher ${ }^{\S}$

Florida State University, Tallahassee FL 32306-4120

\begin{abstract}
The accurate prediction of jet noise is possible only if the jet flow field can be predicted accurately. Predictions for the mean velocity and turbulence quantities in the jet flow field are typically the product of a Reynolds-averaged Navier-Stokes solver coupled with a turbulence model. To evaluate the effectiveness of solvers and turbulence models in predicting those quantities most important to jet noise prediction, two CFD codes and several turbulence models were applied to a jet configuration over a range of jet temperatures for which experimental data is available.
\end{abstract}

\section{Introduction}

The importance of reducing jet noise in both commercial and military aircraft applications has made jet acoustics a significant area of research. ${ }^{1}$ The prediction of jet noise has two primary elements: the prediction of the jet flow field, including turbulence quantities, and the prediction of the acoustic field generated by that turbulent flow field. The objective of the present work is to evaluate the ability of two modern Navier-Stokes flow solvers, coupled with several modern turbulence models, to predict the mean flow and turbulence properties of a supersonic jet plume. This evaluation is accomplished by comparison with experimental jet data, permitting the weaknesses of the flow solvers and models. to be identified. The ultimate goal of this research is to develop a reliable flow solver applicable to the lownoise, propulsion-efficient, nozzle exhaust systems being

\footnotetext{
- Visiting Associate Professor, Department of Mathematics and Program in Computational Science and Engineering, Member AIAA

† Senior Research Scientist, Associate Fellow AIAA

$\ddagger$ Eminent Scholar and Director, Program in Computational Science and Engineering, Member AIAA.

$\$$ Associate Professor, Department of Mathematics and Program in Computational Science and Engineering, Senior Member ALAA

Copyright (C) 1998 by the American Institute of Aeronautics and Astronautics, Inc. No copyright is assented in the United States under Title 17, U.S. Code. The U.S. Govemment has a royalty-free license to exercise all rights under the copyright claimed herein for govemmental purposes. All other rights are reserved by the copyright owner.
}

developed in NASA focused programs. These programs address a broad range of complex nozzle geometries operating in high temperature, compressible, flows.

The development of a reliable flow solver to be applied to noise prediction entails the proper treatment of such issues as gas compressibility, variation of the turbulence dissipation rate due to heat transfer and threedimensionality in turbulent shear flow (i.e., turbulence anisotropy.) In a round-jet application of the MGB acoustics code ${ }^{2}$ for jet-noise prediction, Khavaran ${ }^{3}$ used the PARC aerodynamic code with the two-equation $k-\varepsilon$ turbulence model and standard Launder coefficients ${ }^{4}$ to predict the jet-plume turbulence structure. The acoustic predictions by this Lighthill acoustic-analogy ${ }^{5}$ approach, with the necessary turbulent length and time scales produced by the flow solver, were in reasonable agreement with experimental measurements reported by Yamamoto et al. ${ }^{6}$ However, the extension of this noise-prediction approach to a wide class of nozzle geometries and wide operating ranges of jet total pressure and temperature requires the adequate treatment of turbulent compressibility and anisotropy effects. Such a treatment is the goal of the present research.

Seiner, Ponton, Jansen and Langen ${ }^{7}$ previously discussed the jet configuration examined here. This convergent-divergent nozzle with an exit diameter of 3.6 in. was designed for an exhaust Mach number of 2.0 and a total temperature of $1680^{\circ} \mathrm{F}$. The acoustic and 


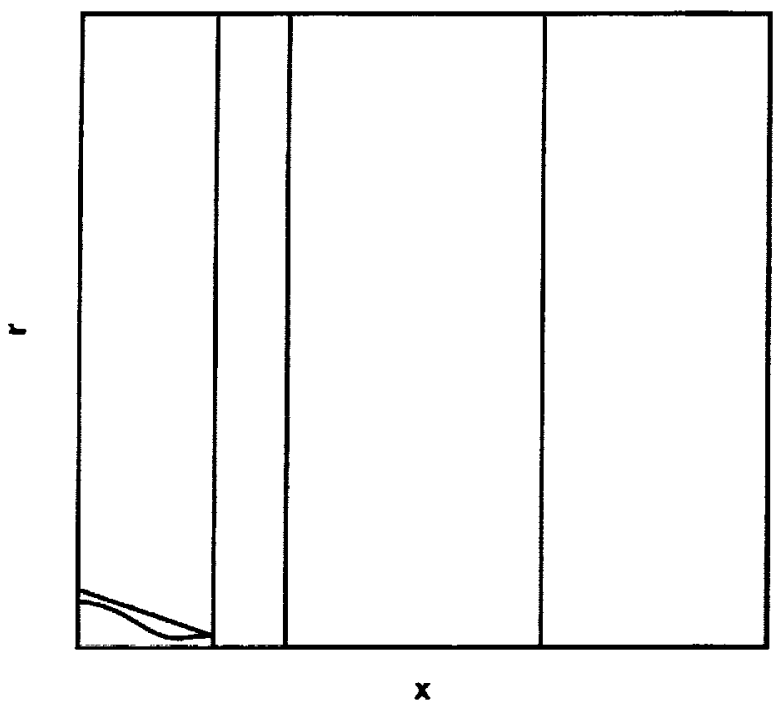

Fig. 1 Computational domain

aerodynamic data reported by Seiner et $a l^{7}$ covered a range of jet total temperatures from $104^{\circ} \mathrm{F}$ to $2200^{\circ}$ $F$ at the fully-expanded nozzle pressure ratio. The aerodynamic data included centerline and radial mean velocity and total temperature profiles.

Computations were performed independently with two CFD codes, ISAAC ${ }^{8}$ and PAB3D. ${ }^{9}$ Turbulence models employed include the $k-\varepsilon$ model, ${ }^{10}$ the Gatski-Speziale algebraic-stress model, " the Girimaji model $^{12}$ and each of these models with the Sarkar compressibility correction. ${ }^{13}$ Centerline and radial values of such quantities as mean velocity, Mach number and mean temperature are compared with the experimental data.

Overall, the qualitative behavior of the experimental data was captured fairly well by both codes and most models. For the mean quantities compared here, the algebraic-stress models did not always represent an improvement over the $k-\varepsilon$ model and the compressibility correction did not always represent an improvement over the uncorrected models.

\section{Codes and Models}

Both CFD codes used in this investigation were developed at NASA Langley Research Center and are intended for use in the solution of turbulent, viscous, flows (employing turbulence models) about general geometries.

ISAAC (Integrated Solution Algorithm for Arbitrary Configurations) ${ }^{8}$ is a finite-volume code of second-order accuracy which solves the full Navier-Stokes equations. An upwind scheme based on Roe's flux-splitting scheme is applied to the convective terms, diffusion terms are centrally differenced and iterations are performed using an implicit, spatially split, approximate-factorization scheme.
The PAB3D code 9 solves the Reynolds-averaged Navier-Stokes equations with some stream-wise derivatives of the viscous terms neglected. Diffusion terms are discretized by a central-difference scheme. The implicit iteration operator is constructed with the van Leer scheme and the explicit terms (such as the convective terms) are evaluated with the Roe upwind scheme.

The grid used in these simulations is constructed of five blocks, one in the nozzle $(61 \times 61$ mesh points), one external to the nozzle $(61 \times 61$ mesh points) and three blocks downstream of the nozzle exit $(65 \times 121,97 \times 121$ and $97 \times 121$ mesh points). The computational domain is shown in Figure 1. Velocity, pressure and temperature boundary conditions consistent with experimental conditions are imposed at the upstream boundary inside the nozzle and a modest free-stream flow of $M=0.05$ is imposed at all non-wall boundaries except the downstream boundary, where a subsonic outflow boundary condition is imposed. No-slip boundary conditions are imposed at all walls. The flow is assumed to be axisymmetric.

The turbulence models employed in this investigation include both $k-\varepsilon$ and algebraic-stress two-equation models. These models span a range of models usable in practical computations today, from the more well-tested models to newer models whose capabilities are not yet so fully explored. Tests of these models give an accurate picture of the current status of flow-field computations for jet noise prediction.

In all computations, the Favre-averaged compressible Navier-Stokes equations ${ }^{8}$ for the averaged density $\bar{\rho}$ and the velocity components $\tilde{u}_{i}$,

$$
\begin{gathered}
\bar{\rho}_{, t}+\left(\bar{\rho} \tilde{u}_{k}\right)_{, k}=0 \\
\left(\bar{\rho} \bar{u}_{i}\right)_{, t}+\left(\bar{\rho} \bar{u}_{i} \bar{u}_{j}\right)_{, j}=\bar{\sigma}_{i j, j}-\left(\bar{\rho} \tau_{i j}\right)_{, j},
\end{gathered}
$$

are employed, where $\bar{\sigma}_{i j}$ denotes the Favre-averaged viscous stress and commas denote differentiation. The molecular viscosity $\mu$ is determined by Sutherland's law.

The Reynolds stress, $\tau_{i j}$, is modeled by the expression $^{14}$

$$
\begin{aligned}
\bar{\rho} \tau_{i j} & =\frac{2}{3} \bar{\rho} k \delta_{i j}-2 \bar{\rho} C_{\mu}^{*} f_{\mu} \frac{k^{2}}{\varepsilon_{s}}\left[\left(S_{i j}-\frac{1}{3} S_{k k} \delta_{i j}\right)\right. \\
& +\alpha_{4} \frac{k}{\varepsilon_{s}}\left(S_{i k} W_{k j}+S_{j k} W_{k i}\right) \\
& \left.-\alpha_{5} \frac{k}{\varepsilon_{s}}\left(S_{i k} S_{k j}-\frac{1}{3} S_{k !} S_{k l} \delta_{i j}\right)\right]
\end{aligned}
$$

$S_{i j}=(1 / 2)\left(\bar{u}_{i, j}+\bar{u}_{j, i}\right)$ and $W_{i j}=(1 / 2)\left(\bar{u}_{i, j}-\right.$ $\left.\tilde{u}_{j, i}\right)$ being the symmetric and anti-symmetric parts of the mean-velocity-gradient tensor. The coefficients $C_{\mu}^{*}$, $\alpha_{4}$ and $\alpha_{5}$ are determined by the individual turbulence models discussed below.

The turbulent kinetic energy $k$ and the solenoidal part of the dissipation rate $\varepsilon_{s}$ (the total dissipation $\varepsilon=\varepsilon_{s}+\varepsilon_{c}$ 
is the sum of the solenoidal and curl-free dissipations; in the case of no compressibility correction, $\varepsilon_{c}=0$ ) are determined from the conservation equations

$$
\begin{aligned}
(\bar{\rho} k)_{, t} & +\left(\bar{\rho} \tilde{u}_{j} k\right)_{, j}=\bar{\rho} P-\bar{\rho} \varepsilon \\
& +\left(\left(\mu+\bar{\rho} C_{\mu}^{*} f_{\mu} \frac{k^{2}}{\sigma_{k} \varepsilon_{s}}\right) k_{, j}\right)_{, j} \\
\left(\bar{\rho} \varepsilon_{s}\right)_{, t}+\left(\bar{\rho} \bar{u}_{j} \varepsilon_{s}\right)_{, j}=\bar{\rho} C_{\varepsilon 1} f_{1} \frac{\varepsilon_{s}}{k} P-\bar{\rho} C_{\varepsilon 2} f_{2} \frac{\varepsilon_{s} \bar{\varepsilon}}{k} & +\left(\left(\mu+\bar{\rho} C_{\mu}^{*} f_{\mu} \frac{k^{2}}{\sigma_{\varepsilon} \varepsilon_{s}}\right) k_{, j}\right)_{, j}+\chi_{w} .
\end{aligned}
$$

$P=-\tau_{i j} \tilde{u}_{i, j}$ is the turbulence production.

The $k-\varepsilon$ model, the simplest of the models discussed here, has $\alpha_{4}=0, \alpha_{5}=0$ and $C_{\mu}^{*}$ a constant (commonly denoted $C_{\mu}$ ) that takes the value 0.096 in the ISAAC model and 0.09 in the PAB3D model. In the $k$ and $\varepsilon_{s}$ equations in ISAAC, $\sigma_{k}=1.0, \sigma_{\varepsilon}=1.3, C_{\varepsilon 1}=1.44$ and $C_{\varepsilon 2}=1.83$. In $\mathrm{PAB} 3 \mathrm{D}$, the different value $C_{\varepsilon 2}=$ 1.93 is employed. In order to permit integration to the wall, the damping functions

$$
f_{\mu}=\left(1+\frac{3.45}{\sqrt{R_{T}}}\right)\left[1-\tanh \left(-\frac{y^{+}}{115.0}\right)\right]
$$

and $f_{1}=1, f_{2}=1$ are employed in ISAAC, where $y^{+}=\rho y u_{\tau} / \mu$ ( $y$ is normal to the wall and $u_{\tau}$ is the wall shear velocity) and $R_{T}=\bar{\rho} k^{2} / \mu \varepsilon_{s}$. The remaining quantities in the $\varepsilon$ equation are $\bar{\varepsilon}=\varepsilon_{s}-$ $\mu / \rho|\nabla \sqrt{k}|^{2}$ and $\chi_{w}=\exp \left[-\left(R_{T} / 40\right)^{2}\right]\left[-2 \bar{\rho} \varepsilon_{s} \bar{\varepsilon}+\right.$ $\left.1.5 \bar{\rho}\left(\varepsilon_{s}-2 \mu k /\left(\rho y^{+2}\right)\right)-1.5 C_{\varepsilon 1} \varepsilon_{s} P\right] / k$. In PAB3D, a low Reynolds number model is used in blocks 1 and 2 (the blocks adjacent to the nozzle walls) where $f_{\mu}=\exp \left[-3.41\left(1+R_{T}\right)^{-2}\right], f_{1}=1$, and $f_{2}=$ $1-0.3 \exp \left(-R_{T}^{2}\right) . \quad \tilde{\varepsilon}$ is defined as in ISAAC and $\chi_{w}=2 \mu \mu_{T} / \bar{\rho}\left[\Sigma_{i j}\left(u_{i, j j}\right)^{2}+\Sigma_{i j k}\left(u_{i, j k}^{2}\right)\right]$. In blocks 3,4 and 5, a high Reynolds number model is used with $f_{\mu}=f_{1}=f_{2}=1, \tilde{\varepsilon}=\varepsilon_{s}$, and $\chi_{w}=0$.

The Gatski-Speziale algebraic-stress model ${ }^{\text {II }}$ employs the full Reynolds-stress expression (3) with coefficients

$$
\begin{gathered}
C_{\mu}^{*}=\alpha_{1} \frac{3\left(1+\eta^{2}\right)+0.2\left(\eta^{6}+\zeta^{6}\right)}{3+\eta^{2}+6 \eta^{2} \zeta^{2}+6 \zeta^{2}+\eta^{6}+\zeta^{6}}, \\
\alpha_{4}=\frac{g}{2}\left(2-C_{4}\right), \quad \alpha_{5}=g\left(2-C_{3}\right),
\end{gathered}
$$

that depend on the flow quantities

$$
\eta^{2}=\alpha_{2} \frac{k^{2}}{\varepsilon^{2}} S_{i j} S_{i j}, \quad \zeta^{2}=\alpha_{3} \frac{k^{2}}{\varepsilon^{2}} W_{i j} W_{i j}
$$

with

$$
\alpha_{1}=\frac{g}{2}\left(\frac{4}{3}-C_{2}\right), \quad \alpha_{2}=\frac{g^{2}}{4}\left(2-C_{3}\right)^{2}
$$

and

$$
\alpha_{3}=\frac{g^{2}}{4}\left(2-C_{4}\right)^{2}, \quad g=\left(\frac{1}{2} C_{1}+C_{5}-1\right)^{-1}
$$

The model for the pressure-strain correlation of Speziale, Sarkar and Gatski ${ }^{15}$ is employed to yield the values $C_{1}=6.8, C_{2}=0.36, C_{3}=1.25, C_{4}=0.40$ and $C_{5}=1.88$. No wall damping of the Reynolds stress is required, so $f_{\mu}=0$. In ISAAC, the $\varepsilon$ equation is changed to give proper log-law behavior with the new stress relationship; ${ }^{14}$ the expressions $f_{1}=1, f_{2}=$ $\left(1-\exp \left(-y^{+} / 5.5\right)^{2}, \bar{\varepsilon}=\varepsilon\right.$, and $\chi_{w}=0$ are used in (5). PAB3D employs the same $k$ and $\varepsilon$ equations as are used in the $k-\varepsilon$ model and does not use the sixth-power terms in (7) introduced to make the model agree with rapid-distortion theory in the large-shear limit.

The Girimaji algebraic-stress model ${ }^{12}$ also employs the Reynolds-stress expression of (3), in this case with the coefficients

$$
C_{\mu}^{*}=-\frac{1}{2} G_{1}
$$

and

$$
\alpha_{4}=-\frac{L_{4}}{L_{0}^{1}-\eta_{1} L_{1}^{1} G_{1}}, \quad \alpha_{5}=-\frac{2 L_{3}}{L_{0}^{1}-\eta_{1} L_{1}^{1} G_{1}} .
$$

Here, $\eta_{1}=k^{2} / \varepsilon^{2} S_{i j} S_{i j}$ and

$$
\begin{aligned}
& L_{1}^{0}=\frac{1}{2} C_{1}^{0}-1, L_{1}^{1}=C_{1}^{1}+2 \\
& L_{2}=\frac{1}{2} C_{2}-\frac{2}{3}, L_{3}=\frac{1}{2} C_{3}^{0}-1, \\
& L_{4}=\frac{1}{2} C_{3}^{0}-1 .
\end{aligned}
$$

The factor $G_{1}$ is the (explicit) solution of a cubic algebraic equation; see the original work of Girimaji ${ }^{12}$ for details of this solution and of the selection of the physically relevant root. The PAB3D implementation of this model again employs the $k-\varepsilon$ model $\varepsilon$ equation. Other than the PAB3D version of this model implemented as part of this work (see below), this model has not been implemented in ISAAC.

The Sarkar compressibility correction ${ }^{13}$ provides for the computation of the compressible part of the turbulent dissipation $\varepsilon_{c}$ in terms of the turbulent Mach number $M_{t}=\sqrt{\tau_{i i}} / a_{\infty}$ and the solenoidal dissipation $\varepsilon_{s}$ :

$$
\varepsilon_{c}=\alpha_{c} M_{t}^{2} \varepsilon_{s}
$$

The constant is here taken to be $\alpha_{c}=\mathbf{0 . 5}$.

\section{Results and Discussion}

Computations were performed at jet total temperatures of $104^{\circ} \mathrm{F}$ and $1550^{\circ} \mathrm{F}$. Runs were typically made with the grid coarsened by a factor of 2 or 4 in each direction, 

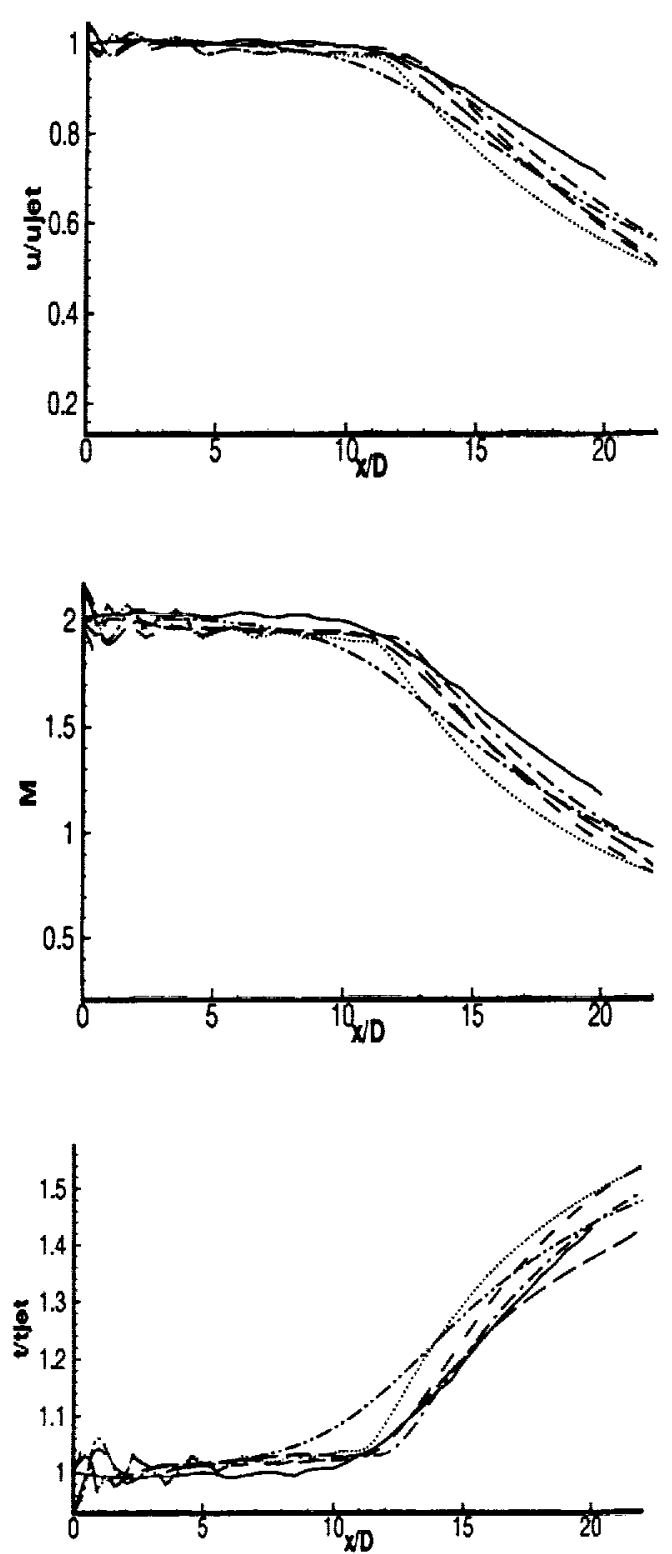

Fig. $2104^{\circ} \mathrm{F}$ case, no compressibility correction. Experiment, solid line; ISAAC $k-\varepsilon$ model, dash-dot-dot line; ISAAC GS ASM, long-dash line; PAB3D $k-\varepsilon$ model, dotted line; PAB3D GS ASM, dash-dot line; PAB3D Girimaji ASM, dashed line.

but runs on the full grid were also performed to confirm the grid-independence of the results. All runs were continued well past the point where no changes at the level of graphical accuracy were seen over an extended period of iteration.

The variation along the centerline of the mean velocity, Mach number and temperature as computed by the models intrinsic to ISAAC and PAB3D are shown in Figures 2-5. It is seen that, in most cases, agreement is reasonable for at least some of the runs. Oscillations
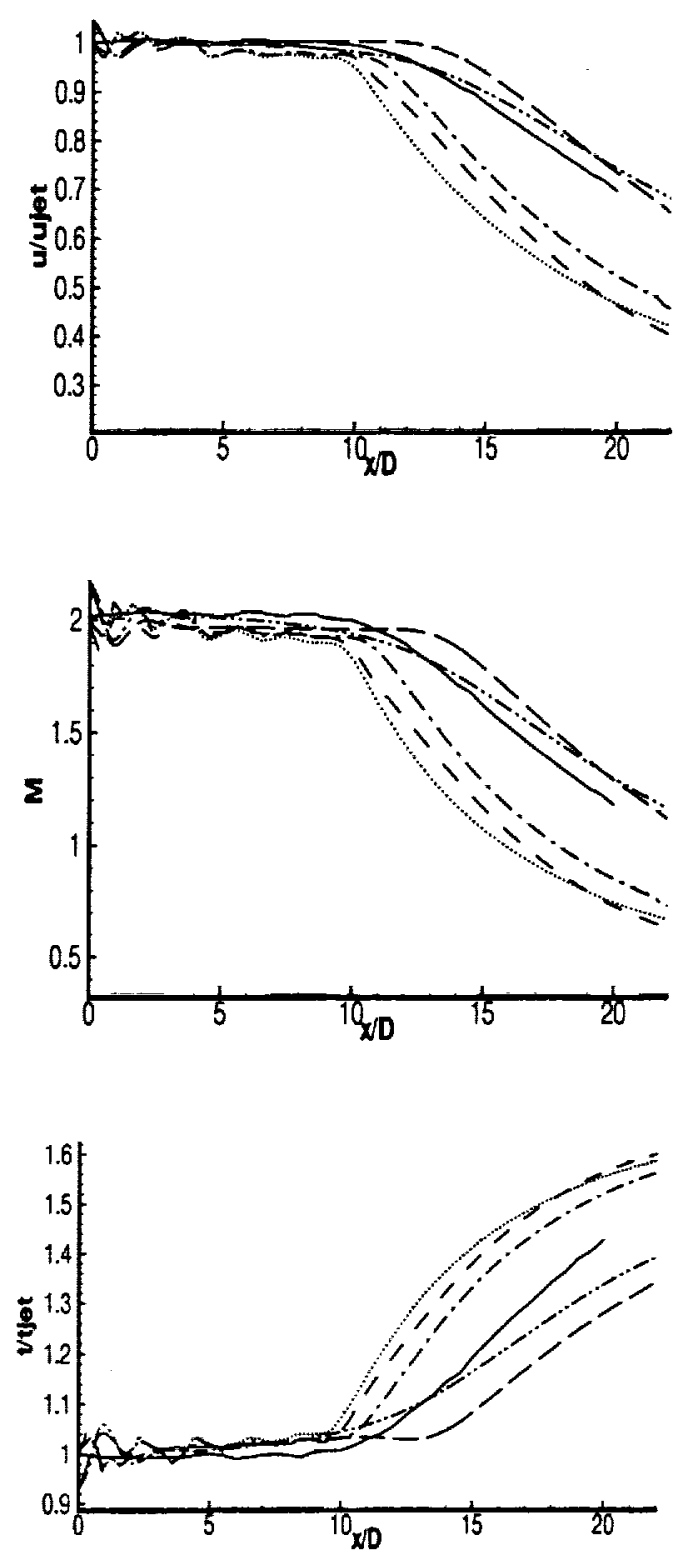

Fig. $3104^{\circ}$ F case, Sarkar compressibility correction. Lines as in Figure 2.

in the velocity and temperature near the nozzle exit are present in both the experimental and numerical results. Schlieren data presented by Seiner et al. ${ }^{7}$ show a weak train of shock waves in the plume; this is normal for a nozzle designed for a single jet total temperature. The numerical simulations, performed in a computational domain that extends into the nozzle interior, also show weak shocks and these are likely to be the cause of the oscillations. Except for details of these oscillations, all runs gave essentially identical results - in good agreement with experiment - within the potential core (the region of essentially inviscid flow downstream of the nozzle between the shear layers emanating from the 
nozzle walls.) The prediction of the end of the potential core region, on the other hand, is a matter of some delicacy, involving as it does the accurate prediction of the viscous and turbulent physical mechanisms of the shear layers over the entire distance from the nozzle exit to the eventual coalescence of the shear layers at the centerline. This prediction, not surprisingly, varies in the results for the different codes and models discussed here. The results downstream of the coalescence of the shear layers, while exhibiting some offset (due, presumably, to misprediction of the coalescence point), have approximately the correct slope in most cases. An interesting additional discrepancy between experiment and computation is the abrupt departure at the end of the potential core from the potential core values in many of the computational results, contrasted with the smoother transition seen in the experiments. While there is no clear superiority of the algebraic-stress models in the results shown here, it should be kept in mind that a comparison of the details of boundary and shear layers could well show advantages of the ASM's.

The Sarkar compressibility correction does not represent a universal improvement in the simulations reported here. In the $104^{\circ} \mathrm{F}$ jet case, the correction makes the PAB3D predictions significantly worse, and the ISAAC predictions are slightly high by about as much as they were low for the computations without the compressibility correction. In the $1550^{\circ} \mathrm{F}$ jet case, the compressibility correction significantly improves the PAB3D results, but makes the ISAAC results dramatically worse.

Despite the generally good agreement between the present results and experiment, there are noticeable differences between the results of the two codes and the different models used in the codes. For the most part, Figures 2-5 show a grouping of the results with ISAAC and its different models and of PAB3D and its different models. This fact suggest the differences in results may be due more to differences in the codes than to differences in the models. In order to further investigate these differences, the $k-\varepsilon$ model in PAB3D was implemented in ISAAC (except for the PAB3D form for $\chi_{w}$, which involves derivatives not readily available in the ISAAC code), as were the PAB3D implementation of the Gatski-Speziale and Girimaji ASM's. Comparisons between the ISAAC implementations of the models, the PAB3D implementations of the models and the PAB3D models implemented in ISAAC are shown in Figures 6,7 and 8 for the $104^{\circ} \mathrm{F}$ case (with no compressiblity correction.) The results from ISAAC with its own models and with the PAB3D models were very similar for the $k-\varepsilon$ model and the Gatski-Speziale algebraicstress model, indicating that the differences between the ISAAC and PAB3D results are primarily due to the codes themselves. The Girimaji ASM had not been previously
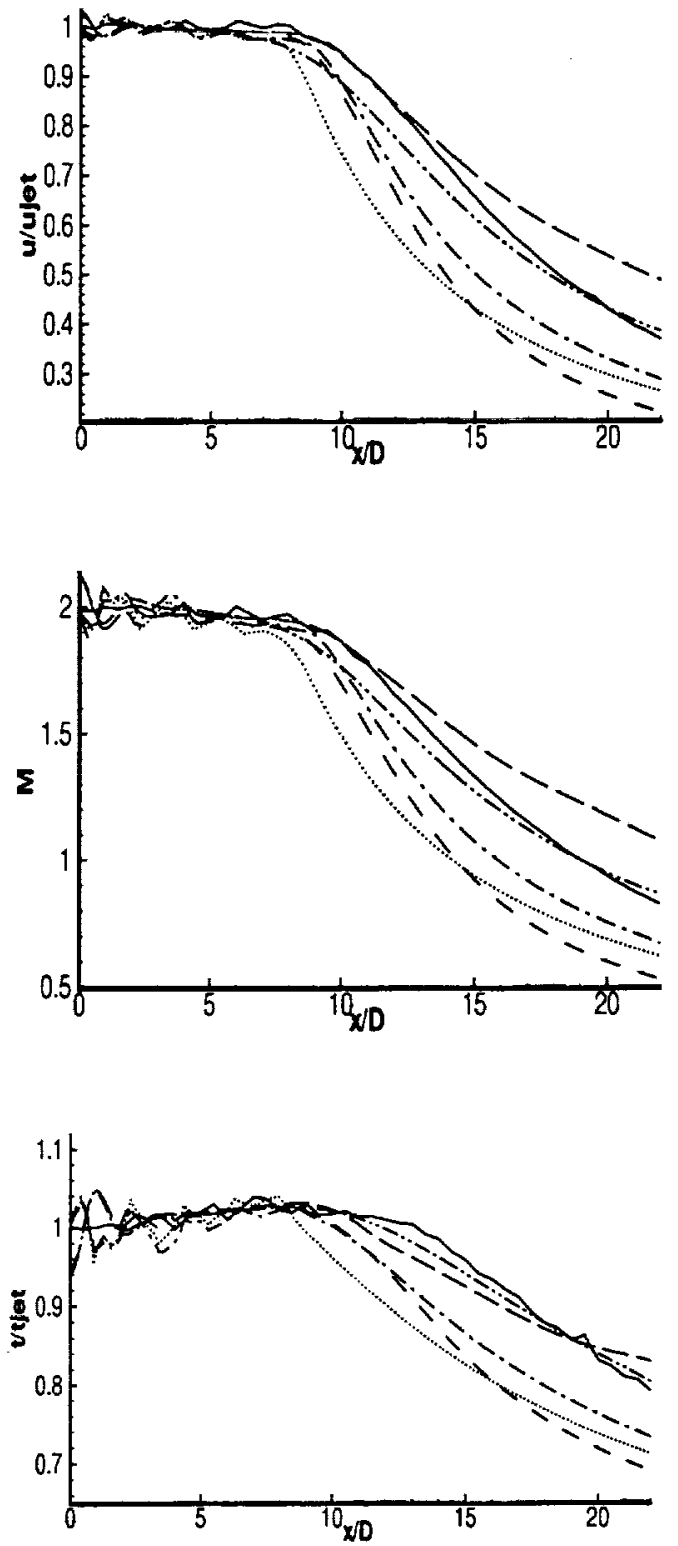

Fig. $41550^{\circ} \mathrm{F}$ case, no compressibility correction. Lines as in Figure 2.

implemented in ISAAC, so Figure 8 contains results only from PAB3D and from the PAB3D implementation of the Girimaji ASM in ISAAC. Curiously, these results are significantly closer than the PAB3D and ISAACwith-PAB3D-model results for the $k-\varepsilon$ model and the Gatski-Speziale algebraic-stress model. An interesting effect encountered in the implementation of the PAB3D models in ISAAC was the convergence to a completely unphysical solution due to the differences in the flowfield intialization of the two codes. A different initialization was required in order to make ISAAC with the PAB3D models converge to the physical solution.

Finally, radial experimental data was available for a 

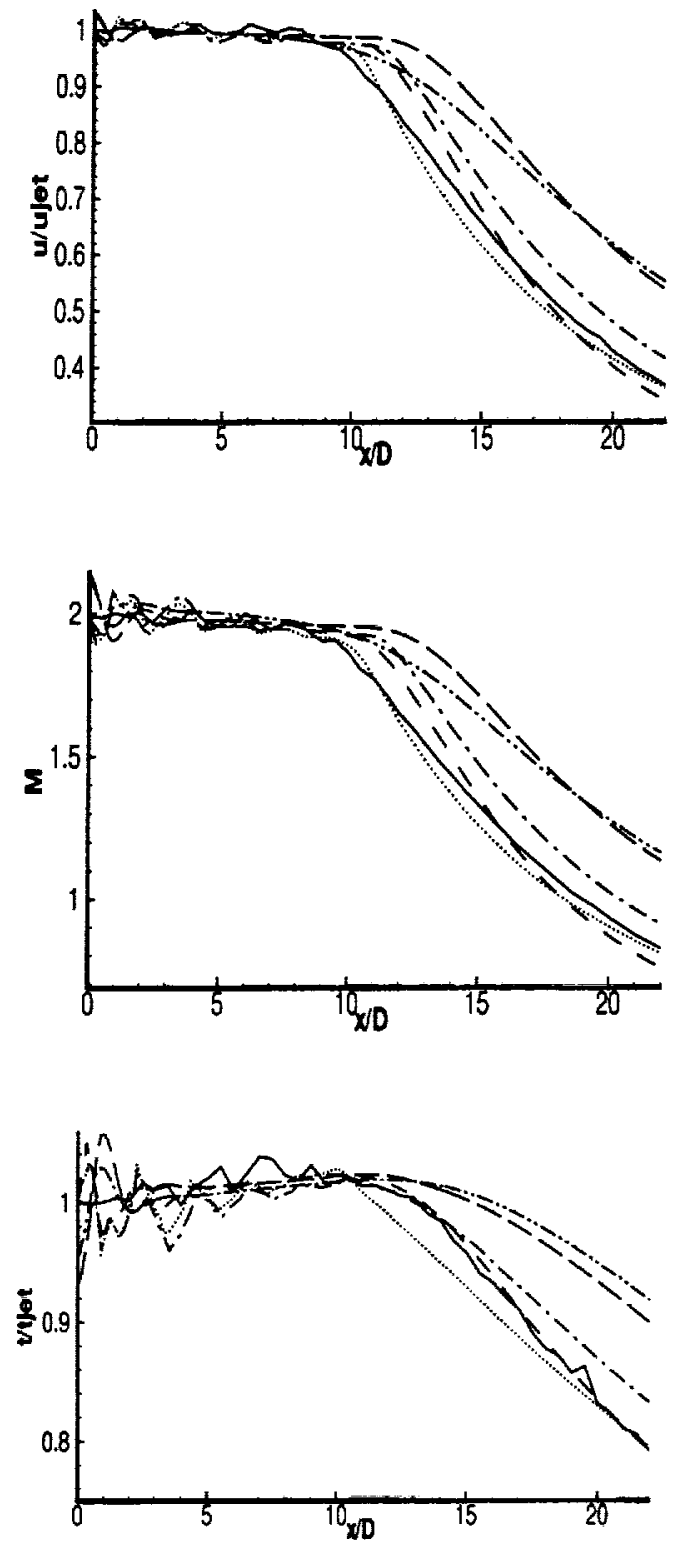

Fig. $51550^{\circ} \mathrm{F}$ case, Sarkar compressibility correction. Lines as in Figure 2.

$900^{\circ} \mathrm{F}$ case, and a comparison between this data and the computational predictions at several positions downstream of the nozzle exit are shown in Figure 9. The predictions of ISAAC and PAB3D are very similar, differing primarily near the centerline, where differences have already been exhibited in the earlier Figures. The predictions are in reasonable agreement with the experimental results.

In summary, overall agreement of the computations with experiment is good. The two codes each gave fairly consistent results with the different turbulence models, and the differences between the codes seemed to be greater than the differences between the models. Addi-
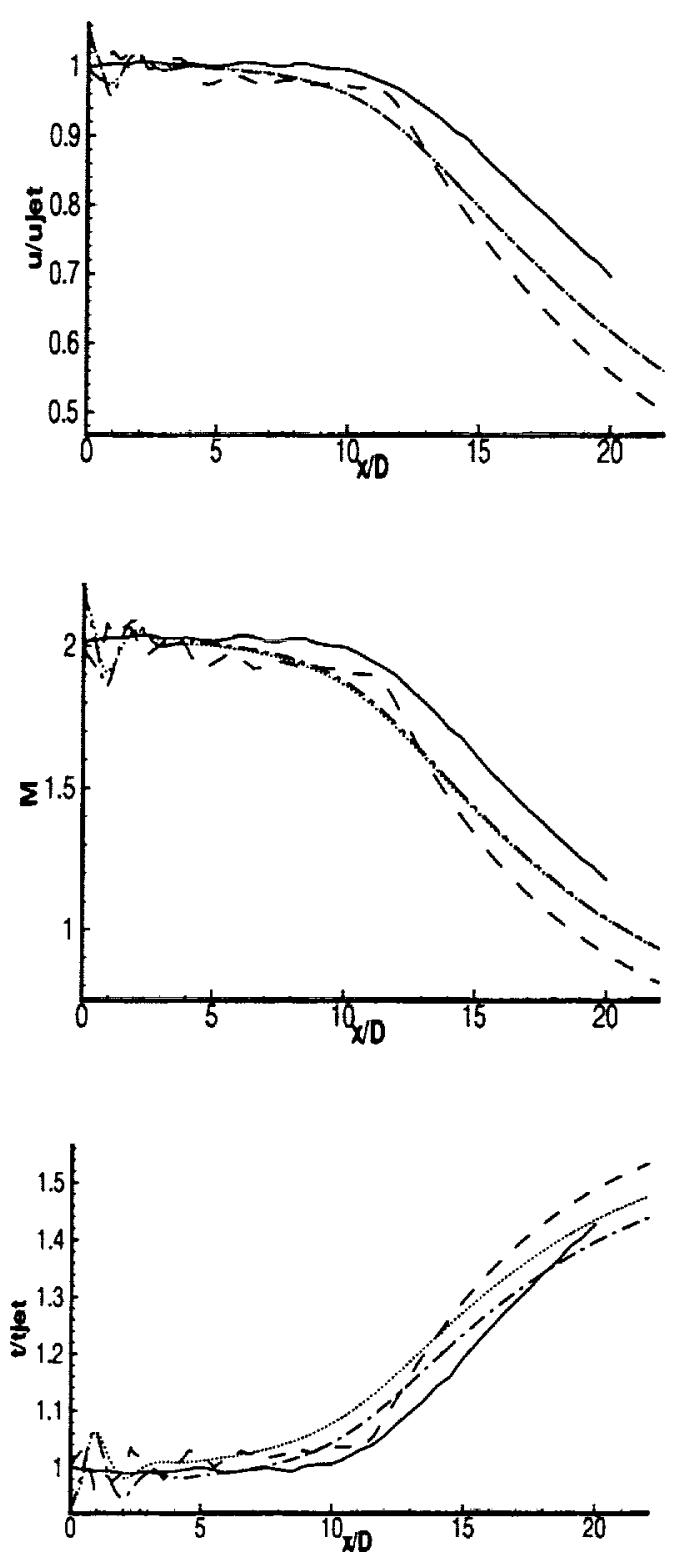

Fig. $6104^{\circ} \mathrm{F}$ case, comparison of $\mathrm{k}-\varepsilon$ models. Experiment, solid line; ISAAC, dash-dot line; PAB3D, dashed line; PAB3D model in ISAAC, dotted line.

tional evidence for this was given by the computations with ISAAC using the PAB3D versions of the models, which gave results much closer to the ISAAC results with its own models than to the PAB3D results. Possible rea sons for these differences between the results of the two codes include different handling of viscous fluxes, first vs. second order advection in the turbulent equations and other differences in the numerical algorithms employed in the two cases. These differences are currently under investigation, as are improvements to the representation of the physics of the problem, such as the incorporation of a variable Prandtl number. 

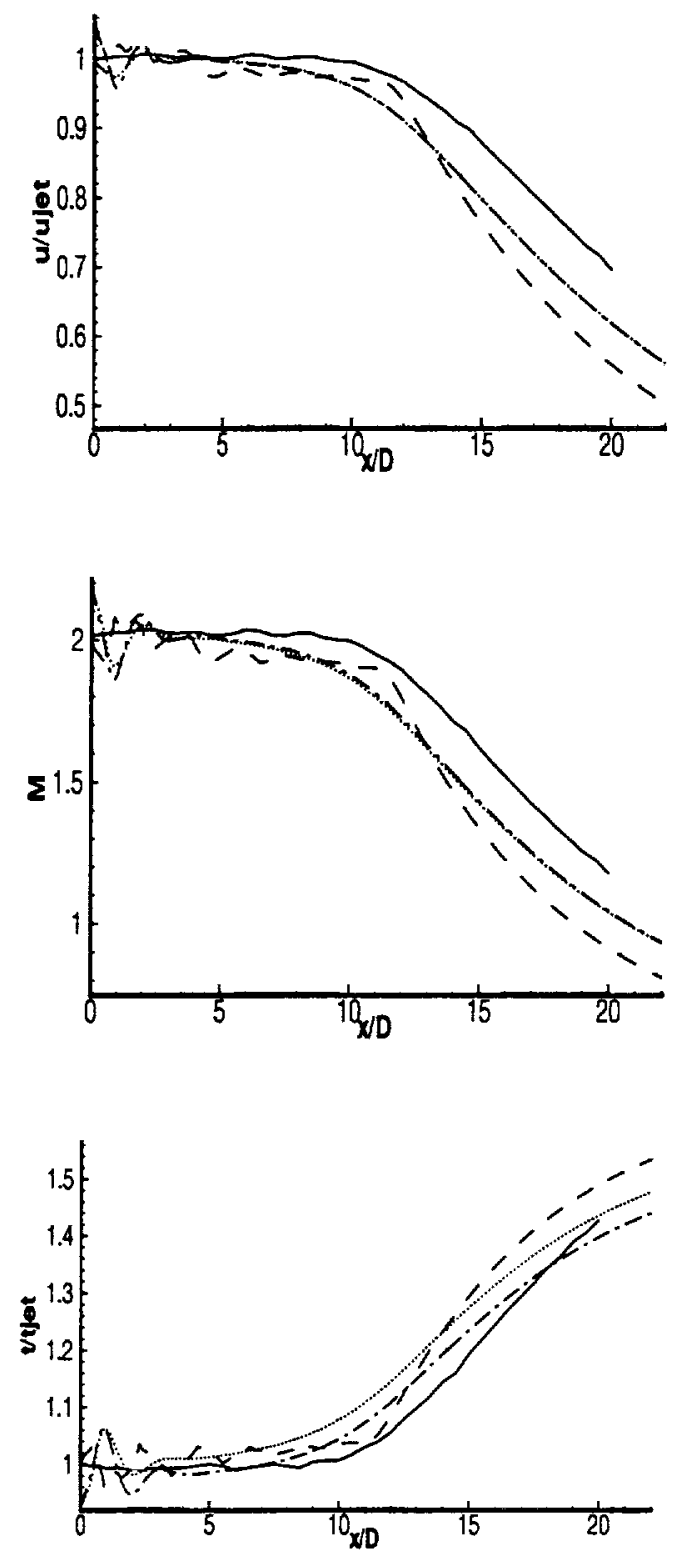

Fig. $7104^{\circ}$ F case, comparision of Gatski-Speziale ASMs. Lines as in Figure 6.

\section{Acknowledgements}

The assistance of J. H. Morrison with ISAAC and P. Pao, J. Carlson and K. S. Abdol-Hamid with PAB3D is gratefully acknowledged. P. Pao and J. Carlson supplied the grid. The ISAAC and PAB3D CFD codes were made available by NASA Langley Research Center. This work was supported by NASA through NASA Langley Research Center.

\section{References}

${ }^{1}$ Seiner, J. M., "Jet noise reduction," Special Volume of Theoretical and Compusational Fluid Dynamics in Honor of Sir James Lighthill, in press, 1997.
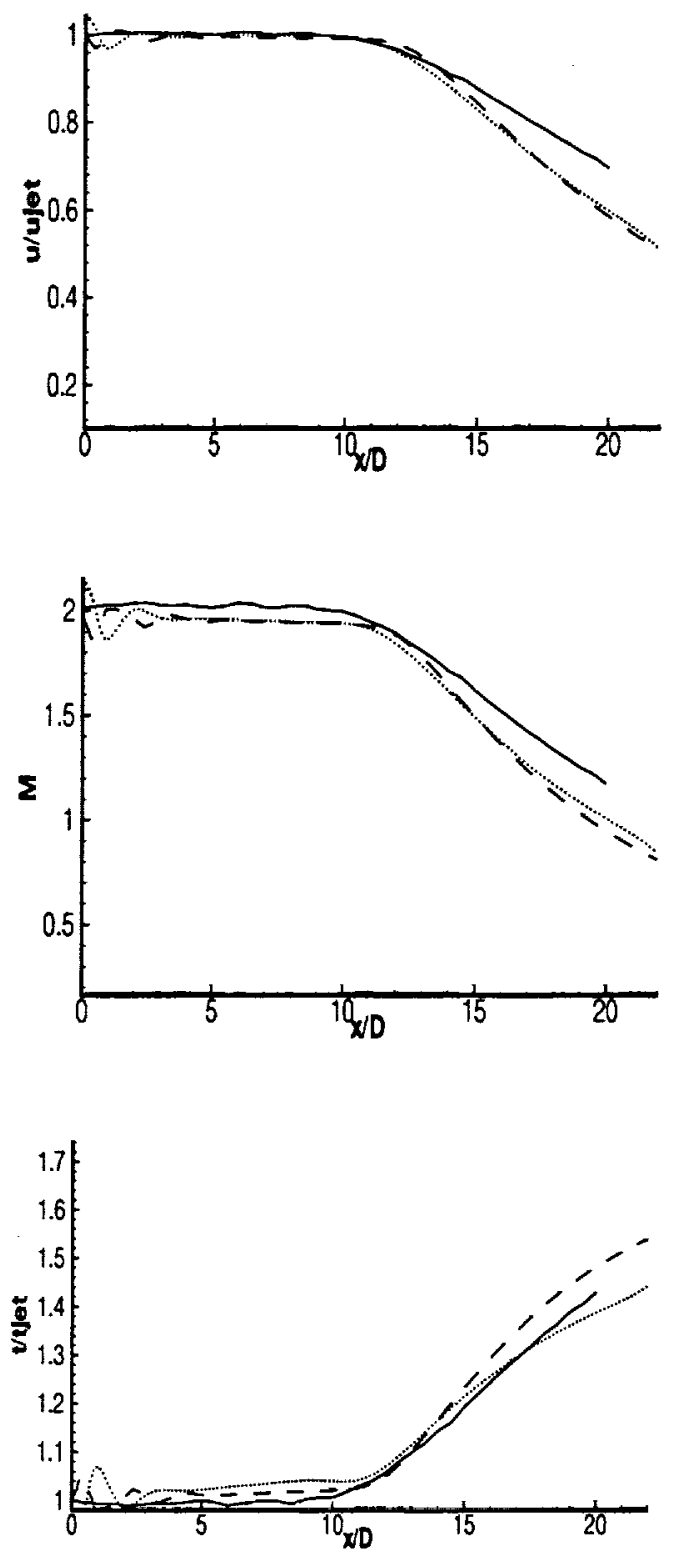

Fig. $8104^{\circ} \mathrm{F}$ case, comparision of Girimaji ASMs. Lines as in Figure 6.

${ }^{2}$ Mani, R., et. al. , "High-velocity jet noise source location and reduction," Task 2, FAA-RD-76-79-II, 1977.

${ }^{3}$ Khavaran, A., "Computation of supersonic jet mixing noise for an axisymmetric convergent-divergent nozzle," AIAA J. Aircrafi 31, 603612

${ }^{4}$ Chien, K. Y., "Prediction of channel and boundary layer flows with a low Reynolds-number turbulence model," AlAA Journal 20, 33-38, 1982.

${ }^{5}$ Lighthill, M. J., “On sound generated aerodynamically. I. General theory." Proc. $R$. Soc. Lond. A211, 564587, 1952.

GYamamoto, K., Brausch, J. F., Balsa, T. F., Janardan, B. A. and Knou, P. R., "Experimental investigation of shock-cell noise reduction for single stream nozzles in simulated flight," NASA CR-3845, 1984.

${ }^{7}$ Seiner, J. M., Ponton, M. K., Jansen, B. J. and Lagen, N., "The effects of temperature on supersonic jet noise emission," DGLR/AIAA 

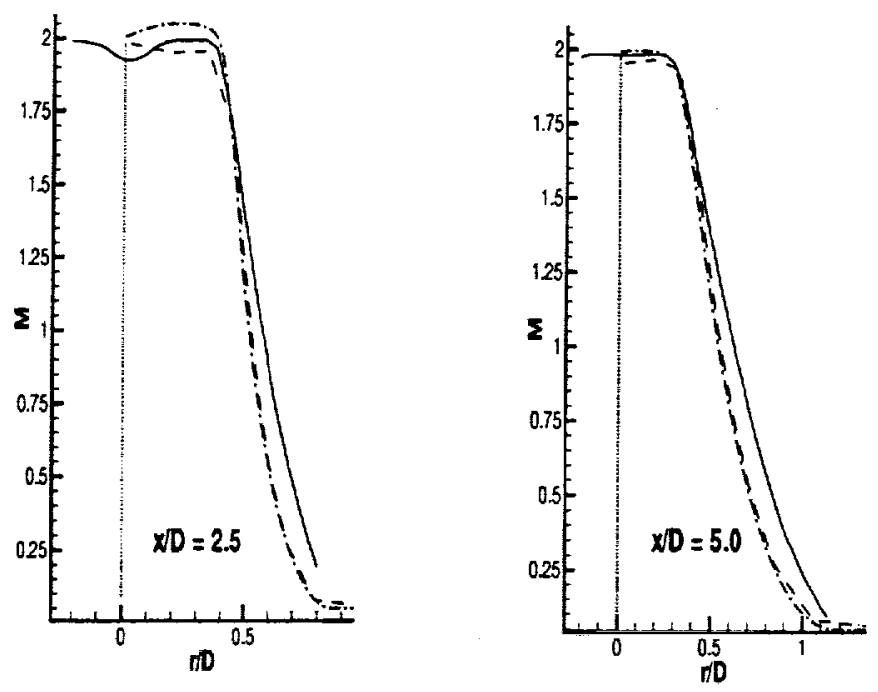

"The analysis and modelling of dilatational terms in compressible turbulence," J. Fluid Mech., 227, 473-493, 1991.

${ }^{14}$ Abid, R., Morrison, J. H., Gatski, T. B. and Speziale, C. G., "Prediction of complex aerodynamic flows with explicit algebraicstress models," AIAA 96-0565, 1996.

${ }^{15}$ Speziale, C. G., Sarkar, S. and Gatski, T. B., "Modeling the pressure-strain correlation of urbulence: an imariant dynamicalsystems approach," J. Fluid Mech. 227, 245-272, 1991.
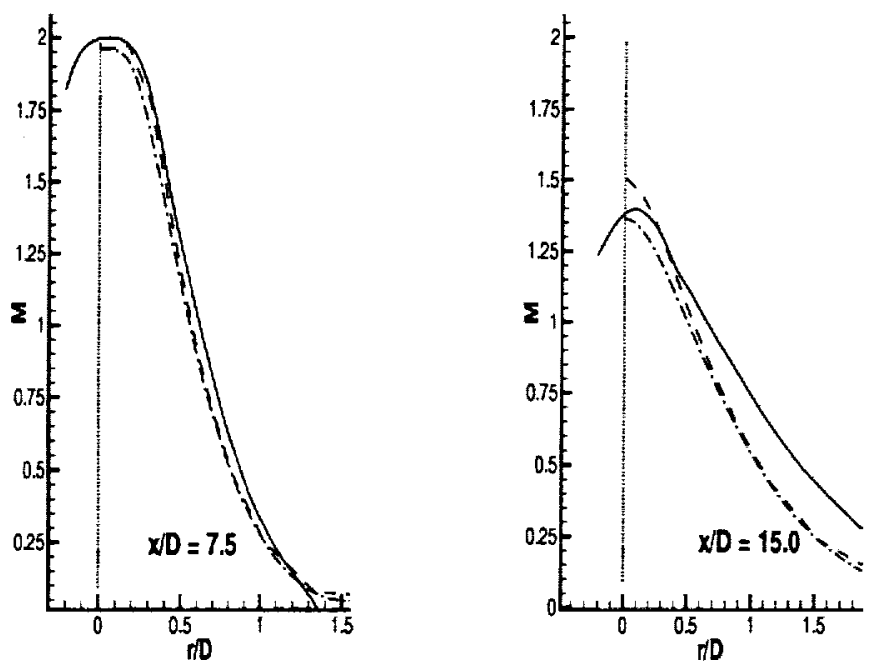

Fig. $9900^{\circ} \mathrm{F}$ case, radial profiles. Experiment, solid line, ISAAC, dash-dotted line; PAB3D, dashed line.

14th Aeroacoustics Conference, Aachen, Germany, Paper No. 92-02. 046

${ }^{8}$ Morrison, J. H., "A compressible Navier-Stokes solver with twoequation and Reynolds-stress turbulence closure models," NASA CR $4440,1992$.

${ }^{9}$ Abdol-Hamid, K. S., "Implementation of algebraic-stress models in a general 3-d Navier-Stokes method (PAB3D)," NASA CR 4702, 1995.

${ }^{10}$ Wilcox, D. C., Turbulence Modeling for CFD, DCW Industries, Inc., LaCanada, CA, 1993.

${ }^{11}$ Gatski, T. B., and Speziale, C. G., "On explicit algebraic-stress models for complex turbulent flows," J. Fluid Mech., 254, 59-78, 1993.

${ }^{12}$ Girimafi, S. S., "Fully-explicit and self-consistent algebraic Reynolds-stress model," ICASE Report 95-82, 1995.

${ }^{13}$ Sarkar, S., Erlebacher, G., Hussaini, M. Y. and Kreiss, H.O.,

8 OF 8 